\section{Tools for studying early events in optic neuropathies}

\begin{abstract}
Most optic nerve injuries are axonal. Neurotrophin deprivation induces death of retinal ganglion cells (RGCs) in such cases, but this is probably not the only mechanism that causes their death. Various fluorophores and agents that block mitochondrial electron transport have been used to show that superoxide generated in the mitochondrial electron transport chain could act, in addition to neurotrophin deprivation, to signal cell death after axonal injury. More specifically, sulphydryls - probably on proteins - are downstream regulators of this signalling pathway, as shown by the neuroprotective effects of inhibition of sulphydryl oxidation by tris(2-carboxyethyl)phosphine in in vivo rat models. Other tools used to study early events in RGC death include novel reducing agents, inducible superoxide dismutase, and differentiation of the RGC-5 cell line.
\end{abstract} Eye (2007) 21, S21-S24; doi:10.1038/sj.eye.6702883

Keywords: retinal ganglion cells; axonal injury; apoptosis; superoxide; reactive oxygen species

\section{Introduction}

Most optic nerve injuries are axonal, which lead to the death of retinal ganglion cells (RGCs). After axotomy, RGCs undergo neurotrophin deprivation, but this mechanism alone may not be enough to cause their death. A specific reactive oxygen species (ROS), superoxide anion, may act as an intracellular messenger for signalling RGC death, as an additional mechanism. ${ }^{1}$ Research suggests critical sulphydryls (molecular groups that include a bonded sulphur and hydrogen atom), probably on proteins, may be downstream regulators of this signal pathway. ${ }^{2}$ Monitoring the levels of these two potential surrogate biomarkers of RGC apoptosis could be a
CJ Lieven ${ }^{1}$ and LA Levin ${ }^{1,2}$

useful tool to detect early events in optic neuropathies.

Role of reactive oxygen in neuronal cell death

Oxygen-induced retinal ganglion cell death

Certain types of cell death require oxygen, and this can be shown in axotomized RGCs in culture. ${ }^{2}$ Hypoxia inhibits this death; the cells die faster when cultured in oxygen than in nitrogen. The natural environment of RGCs is low in oxygen, with a partial pressure of oxygen in the RGC layer of about $20 \mathrm{mmHg}^{3}$ This is much lower than in the air we breathe or the partial pressure in most tissue culture incubators. It is also lower than in the environment at the outer segments of the photoreceptor, but higher than that at the inner segments.

Reactive oxygen species act as an early signal for neuronal apoptosis

In cultured sympathetic neurons undergoing neurotrophin deprivation, apoptosis is delayed when superoxide dismutase (SOD) is injected or overexpressed. ${ }^{4}$ However, when the SOD is injected $8 \mathrm{~h}$ after neurotrophin deprivation, this anti-apoptotic effect is not seen. In this case, the increase in ROS peaks at $3 \mathrm{~h}$ after the neurons are deprived of the nerve growth factor on which sympathetic neurons are highly dependent. When the growth factor is added back after this peak time, apoptosis is completely prevented. The researchers interpreted this to mean that ROS production is an early signal for neuronal apoptosis. These findings raised the possibility of parallels in RGCs, which became a focus of the research described below.

Fluorescence imaging of retinal ganglion cells in vitro

Intracellular levels of superoxide anion in cultured rat RGCs have been measured using
${ }^{1}$ Department of

Ophthalmology and Visual Sciences, University of Wisconsin Medical School Madison, WI, USA

${ }^{2}$ Department of Ophthalmology, University of Montréal, Montreal, Quebec, Canada

Correspondence: LA Levin, Department of Ophthalmology and Visual Sciences,

University of Wisconsin Medical School, 600 Highland Avenue, Madison, WI 53792, USA Tel: + 16082656546 . 
fluorophores that are highly sensitive to this molecule. ${ }^{1}$ Two different probes were used, of which hydroethidium is one of the more commonly used. In the presence of superoxide, it forms a moiety, oxy-ethidium, which binds to DNA and changes its emission spectrum. Using this method, it was possible to show a significant increase in the level of superoxide on the scale of individual RGCs (Figure 1).

\section{Superoxide rise in axotomized retinal ganglion cells}

There is a slow, asynchronous rise in superoxide in RGCs after axotomy, reflecting the slow rate at which RGCs die after axonal injury (Figure 2). ${ }^{1}$ In the rat, for example, only half of the RGCs die over 7 days after axotomy, and this asynchronous and slow death rate occurred even when there was very abrupt injury, such as the complete transection of all axons. ${ }^{5}$ The superoxide increase was not just a phenomenon related to dissociation, but a result of the axotomy. ${ }^{1}$ By the end of 1 week after axotomy, mean superoxide levels in the remaining RGCs started to shift back to normal levels. The rise after axotomy was not blocked by neurotrophic factors and cyclic AMP and therefore appeared to be at least partially independent of these factors (ie not caused by neurotrophin deprivation). ${ }^{1}$

Among several inhibitors of intracellular superoxide generation studied, only antimycin A, which inhibits complex III of the mitochondrial electron transport chain, blocked the increase in superoxide. This finding demonstrates that the superoxide is generated in the mitochondrial electron transport chain, and that it could act in parallel to neurotrophic deprivation for signalling cell death after axonal injury.

\section{Role of sulphydryl reduction in neuronal apoptosis}

It is believed that ROS cause cell death by influencing the redox state of the RGC. ${ }^{2}$ Other groups have also shown that RGCs have an optimum redox state that is slightly reduced just after axotomy, if they survive. ${ }^{6}$ This suggests that one or more sulphydryls are oxidized, for example, by superoxide, and the cells then die. Consequently, by reducing critical oxidatively modified sulphydryls, RGCs can be saved.

In vitro, it has been possible to show that reduction protects axotomized RGCs in the absence of neurotrophic factors. Axotomized RGCs usually survive about 3-6 days in culture. If suphydryl oxidation is blocked or reversed, the cells survived up to 2 weeks (Figure 3 ). ${ }^{2}$ In vivo, intravitreal injection to animal models of a specific agent, tris(2-carboxyethyl)phosphine, also block suphydryl oxidation, and prolong RGC survival. ${ }^{7}$ There may thus be suphydryl oxidation of one or more critical moiety, leading to an apoptosis signal. The target of this signal is not known and might be a component of the mitochondrial permeability transition pore, a ribonuclease inhibitor, or an unknown target(s) causing zinc release from intracellular stores.

\section{Other tools for studying early events in retinal ganglion cell apoptosis}

Various other tools have been developed to study early events in RGC apoptosis. The novel reducing compounds PB1 and PB2 were designed using a pharmacological approach to increase their efficacy in improving RGC viability. These modified neuroprotective molecules potentially target intracellular
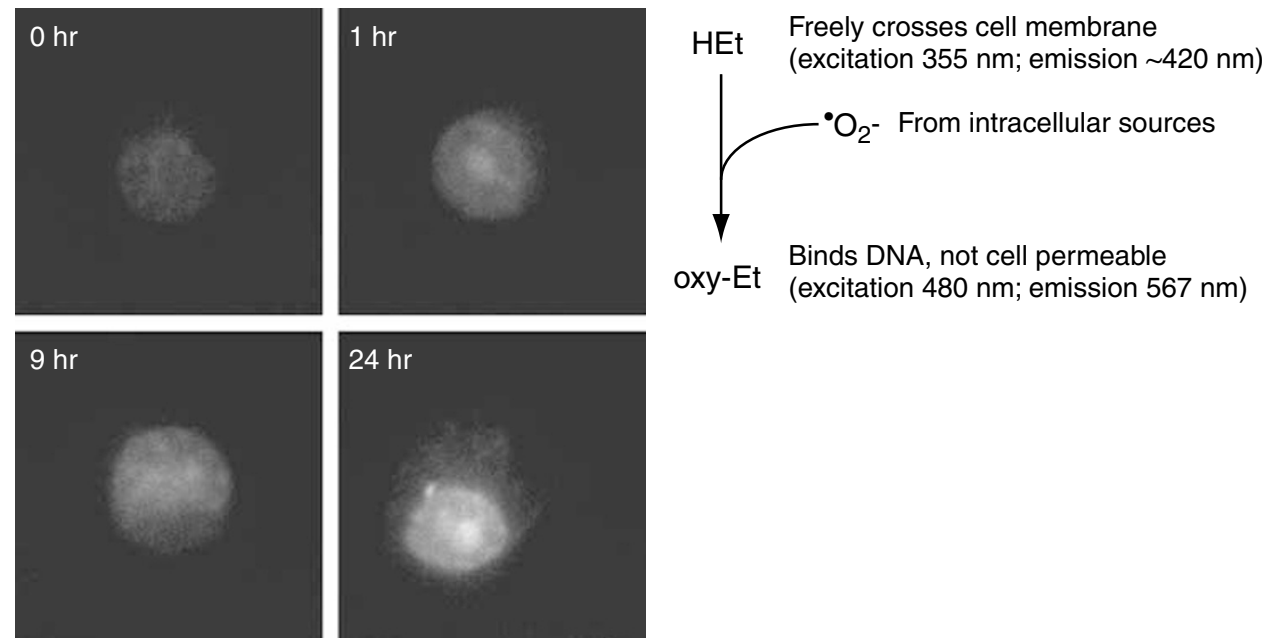

Figure 1 Fluorescence imaging of retinal ganglion cells using hydroethidium as a superoxide probe: the level of superoxide over time, as seen by the level of emission, can be seen. (Reproduced from Lieven et al ${ }^{1}$ with permission of Invest Ophthalmol Vis Sci via Copyright Clearance Center.) 
Menadione
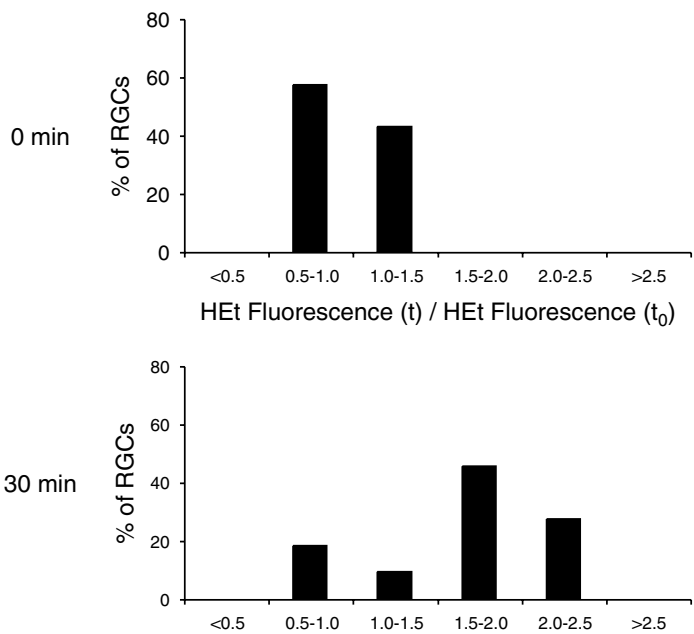

HEt Fluorescence (t) / HEt Fluorescence $\left(\mathrm{t}_{0}\right)$

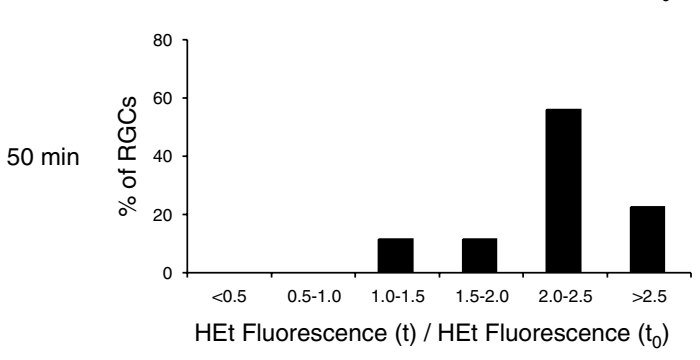

HEt Fluorescence ( $\mathrm{t}) / \mathrm{HEt}$ Fluorescence $\left(\mathrm{t}_{0}\right)$

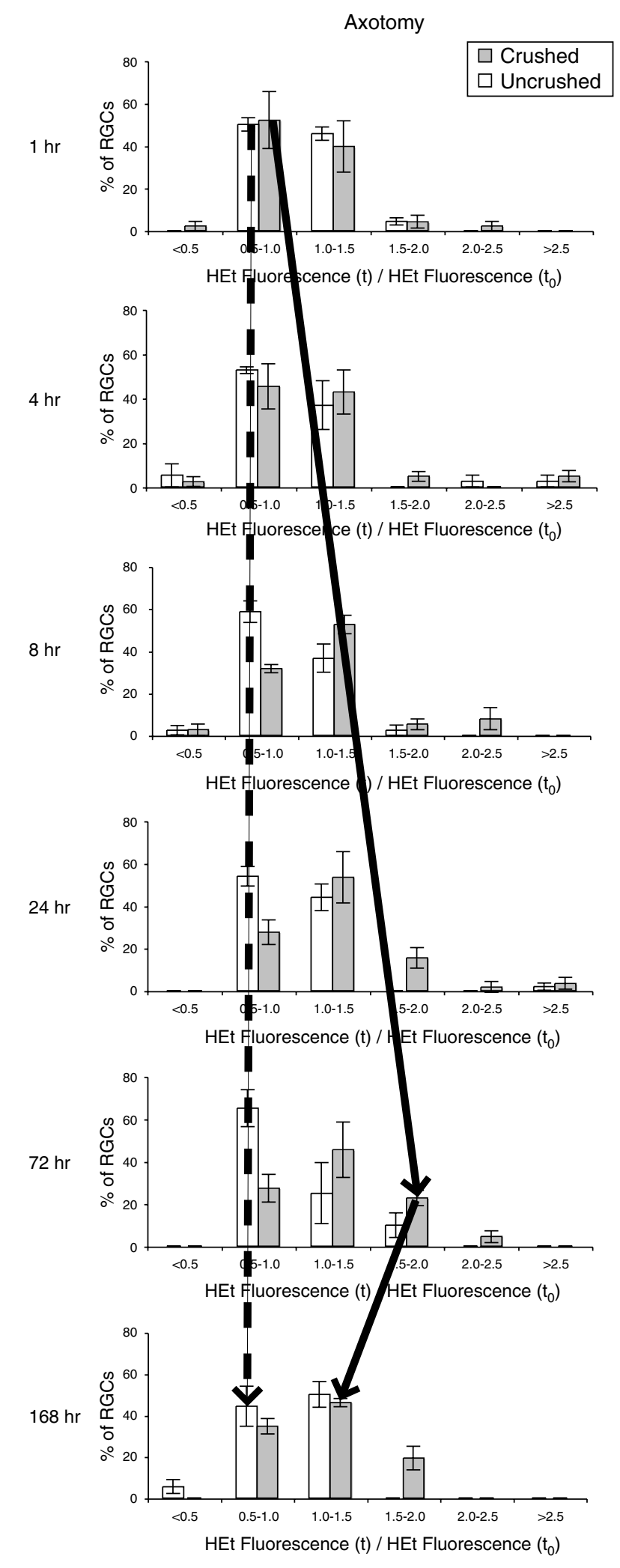

Axotomy

$1 \mathrm{~h}$

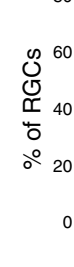

Figure 2 Slow and asynchronous superoxide increase over time in cultured RGCs after axotomy: compare with the rapid and steep rise obtained with menadione, which is a drug that increases superoxide artificially. (Reproduced from Lieven et al $^{1}$ with permission of Invest Ophthalmol Vis Sci via Copyright Clearance Center.) 


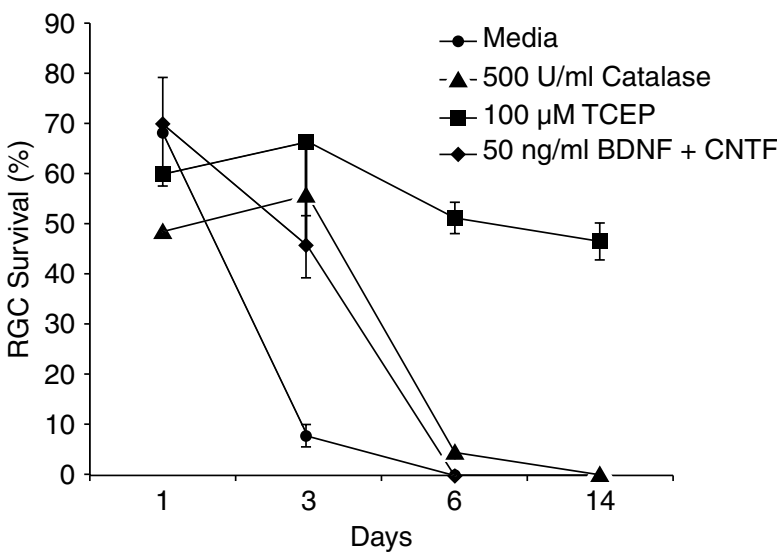

Figure 3 Blocking suphydryl oxidation rescues axotomized RGCs. (Reproduced from Geiger $e t a^{2}$ with permission of Neurosci via Copyright Clearance Center.)

sulphydryls and are highly cell-permeable. As a result of this approach, PB1 is highly active at the nanomolar level, and PB2 is active at the picomolar level. ${ }^{8}$

Early events in RGC apoptosis can also be studied by using a regulated SOD gene under the control of a tetracycline-related promoter. Thus in the future it may be possible to study RGC survival in transgenic mice in the presence and absence of doxycycline as a tool to examine whether superoxide is necessary for signalling cell death in vivo in experimental optic neuropathies.

Another tool is the RGC- 5 cell line, invented by Neeraj Agarwal. ${ }^{9}$ This cell line resembles an RGC in terms of its antigen production, but is morphologically like a glial cell, and does not have ion channels characteristic of RGCs. In the presence of staurosporine, a drug that normally kills cells, RGC- 5 cells do not die, but differentiate into cells that morphologically resemble RGCs, and express neuronal markers characteristic of RGCs. ${ }^{10}$ These differentiated RGC-5 cells do not divide, and are useful for studying different aspects of RGCs development and pathophysiology, particularly axonal and dendritic extension, and mitochondrial physiology.

\section{Conclusion}

A variety of tools are available for studying early changes in RGCs after axonal injury. The loss of RGCs and their axons underlies all optic neuropathies. Understanding the early events in these diseases may eventually lead to new therapies for these blinding diseases.

\section{Acknowledgements}

This study was supported by NIH R01EY12492, Retina Research Foundation, and an unrestricted departmental grant from Research to Prevent Blindness, Inc.

\section{References}

1 Lieven CJ, Schlieve CR, Hoegger MJ, Levin LA. Retinal ganglion cell axotomy induces an increase in intracellular superoxide anion. Invest Ophthalmol Vis Sci 2006; 47: 1477-1485.

2 Geiger LK, Kortuem KR, Alexejun C, Levin LA. Reduced redox state allows prolonged survival of axotomized neonatal retinal ganglion cells. Neuroscience 2002; 109: 635-642.

3 Linsenmeier RA, Braun RD. Oxygen distribution and consumption in the cat retina during normoxia and hypoxemia. J Gen Physiol 1992; 99(2): 177-197.

4 Greenlund LJ, Deckwerth TL, Johnson EM. Superoxide dismutase delays neuronal apoptosis: a role for reactive oxygen species in programmed neuronal death. Neuron 1995; 14: 303-315.

5 Berkelaar M, Clarke DB, Wang YC, Bray GM, Aguayo AJ. Axotomy results in delayed death and apoptosis of retinal ganglion cells in adult rats. J Neurosci 1994; 14(7): 4368-4374.

6 Castagne V, Lefevre K, Natero R, Clarke PG, Bedker DA. An optimal redox status for the survival of axotomized ganglion cells in the developing retina. Neuroscience 1999; 93(1): 313-320.

7 Swanson KI, Schlieve CR, Lieven CJ, Levin LA. Neuroprotective effect of sulfhydryl reduction in a rat optic nerve crush model. Invest Ophthalmol Vis Sci 2005; 46(10): 3737-3741.

8 Schlieve CR, Tam A, Nilsson BL, Lieven CJ, Raines RT, Levin LA. Synthesis and characterization of a novel class of reducing agents that are highly neuroprotective for retinal ganglion cells. Exp Eye Res 2006; 83: 1252-1259.

9 Krishnamoorthy RR, Agarwal P, Prasanna G, Vopat K, Lambert W, Sheedlo HJ et al. Characterization of a transformed rat retinal ganglion cell line. Brain Res $\mathrm{Mol}$ Brain Res 2001; 86(1-2): 1-12.

10 Frassetto LJ, Schlieve CR, Lieven CJ, Utter AA, Jones MV, Agarwal $\mathrm{N}$ et al. Kinase-dependent differentiation of a retinal ganglion cell precursor. Invest Ophthalmol Vis Sci 2006; 47(1): 427-438. 